\title{
Pressure Loads by Gas in an Enclosed Chamber in DYNA3D
}

\author{
J. Lin, D.C. Badders
}

August 8, 2002

U.S. Department of Energy

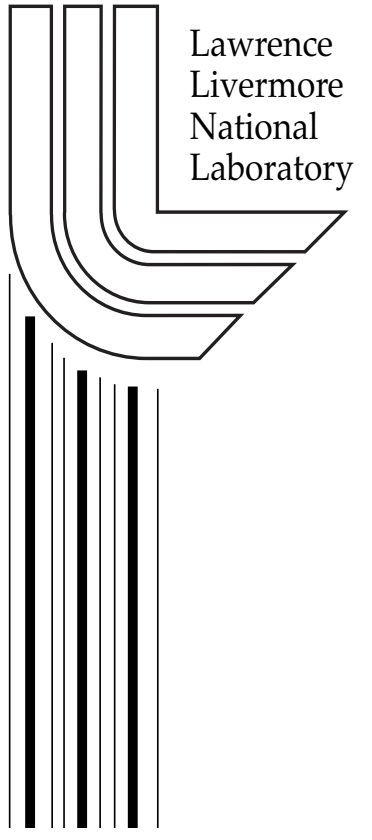




\section{DISCLAIMER}

This document was prepared as an account of work sponsored by an agency of the United States Government. Neither the United States Government nor the University of California nor any of their employees, makes any warranty, express or implied, or assumes any legal liability or responsibility for the accuracy, completeness, or usefulness of any information, apparatus, product, or process disclosed, or represents that its use would not infringe privately owned rights. Reference herein to any specific commercial product, process, or service by trade name, trademark, manufacturer, or otherwise, does not necessarily constitute or imply its endorsement, recommendation, or favoring by the United States Government or the University of California. The views and opinions of authors expressed herein do not necessarily state or reflect those of the United States Government or the University of California, and shall not be used for advertising or product endorsement purposes.

This work was performed under the auspices of the U. S. Department of Energy by the University of California, Lawrence Livermore National Laboratory under Contract No. W-7405-Eng-48.

This report has been reproduced directly from the best available copy.

Available electronically at http://www.doc.gov/bridge

Available for a processing fee to U.S. Department of Energy

And its contractors in paper from

U.S. Department of Energy

Office of Scientific and Technical Information

P.O. Box 62

Oak Ridge, TN 37831-0062

Telephone: (865) 576-8401

Facsimile: (865) 576-5728

E-mail: reports@adonis.osti.gov

Available for sale to the public from

U.S. Department of Commerce

National Technical Information Service

5285 Port Royal Road

Springfield, VA 22161

Telephone: (800) 553-6847

Facsimile: (703) 605-6900

E-mail: orders@ntis.fedworld.gov

Online ordering: http:/ / www.ntis.gov/ordering.htm

\section{OR}

Lawrence Livermore National Laboratory

Technical Information Department's Digital Library

http: / / www.llnl.gov/tid/Library.html 


\section{Pressure Loads by Gas in an Enclosed Chamber in DYNA3D ${ }^{+}$}

By

Jerry Lin and Daniel C. Badders

Defense Technologies Engineering Division

Mechanical Engineering Department Lawrence Livermore National Laboratory

$\downarrow$ This work was performed under the auspices of the U.S. Department of Energy by the University of California, Lawrence Livermore National Laboratory under Contract No. W-7405-Eng-48. 


\begin{abstract}
New algorithms that efficiently calculate the volume of a closed chamber are presented in this paper. The current pressure in the enclosed chamber can then be computed, based on the userspecified gas law, from the updated volume and the initial volume and pressure of the chamber. This pressure load function is very useful in modeling common features, such as air pocket, airbag, piston, and gun barrel, in structural analyses.
\end{abstract}

\title{
1. Introduction
}

In structural analysis of mechanical systems, one often encounters the modeling of gas-filled chamber-like structures. The pressure load imposed on the structure by the enclosed gas will change because of the addition of more gas into the chamber or the change of the chamber volume. The obvious examples for the former are the gun barrel and the vehicle airbag, whereas for the latter the piston chambers in an engine. In computer simulations, the structure surfaces enclosing the gas are usually discretized into triangular or quadrilateral patches. During an analysis, either a pre-defined function of time or a pressure-volume gas law usually determines the gas pressures exerted on these patches. The pre-defined function approach involves predicting the chamber volume change a priori and often does not reflect the interaction between the structure and the gas. The gas law option is usually a more desirable approach since it updates the current chamber volume continuously and thus takes the evolving structural deformation into account.

In an explicit finite element code like DYNA3D [1], because of its inherent limitation on time integration step size, efficient algorithms for updating the chamber volume are essential to the gas law approach. The method we present here hinges on the quick calculation of the volume of truncated triangular prisms formed by the triangular patches that define the closed chamber and their projects on a common plane. Properly selecting the projection plane would make the volume calculation for the truncated prisms trivia. By orienting the triangular patches so that their normal vectors all point outward from the closed chamber, the chamber volume can be quickly obtained by summing the volumes of all truncated prisms.

In structural analysis, symmetry conditions are often utilized to reduce the size of the model. The plane of symmetry may cut through a closed chamber in which the gas pressure is present. In this situation, the chamber would appear open because of the lack of discretized patches on the plane of symmetry to close it. To maintain the effectiveness of the proposed algorithms, the plane of projection must coincide with the plane of symmetry or be perpendicular to it.

The closed chamber such as an airbag is defined by the same set of patches whereas a piston chamber can be defined by different sets of patches depending upon the position of the piston. When the piston motion suppresses the piston chamber, the chamber will be enclosed by fewer patches since some of the patches originally in the list will be entirely covered by the piston. 
These piston-covered patches may later become active again as the piston moves away. A technique adaptively tracking the patches defining the piston chamber is also presented in this paper. It is accomplished by the use of a reference plane moving in the direction of the piston motion.

The truncated prism volume calculation and the summing procedure to get the chamber volume are presented in the next section. Section 3 details the algorithms used to decide the active patches for a piston chamber. Section 4 explains the projection plane selection, including the consideration of planes of symmetry. Pressure calculation and nodal force application is outlined in section 5. Section 6 and section 7 provide the user input sequences and sample problems respectively.

\section{Divergence Theorem of Gauss and the Volume Calculation}

To compute the volume enclosed by an arbitrary piecewise smooth surface, we employ the Divergence Theorem of Gauss [2]. The theorem can be stated as

$$
\iiint_{D} \operatorname{div} \mathbf{u} \mathrm{dV}=\iint_{S} \mathbf{u} \bullet \mathbf{n} \mathrm{dA},
$$

where $D$ is a domain enclosed by a piecewise smooth surface $S$, $\mathbf{u}$ a continuous vector function with continuous first partial derivatives in $D$, and $\mathbf{n}$ the outward unit normal vector of $S$. In finite element analysis, the surface $S$ is usually discretized into finite number of triangular or quadrilateral patches, which lead Eq. (1) to

$$
\iiint_{D} \operatorname{div} \mathbf{u} \mathrm{dV}=\sum_{i=1}^{N} \iint_{S_{i}} \mathbf{u} \bullet \mathbf{n} \mathrm{dA},
$$

where $\mathrm{N}$ is the total number of patches for surface $S$, and $S_{i}$ is the area of the $i$ th patch.

If we choose an appropriate $\mathbf{u}$ such that its divergence is a constant, the summation of surface integrals on the right side of Eq. (2) will then lead to the volume of $D$. One of the obvious choices is the vector field $\mathbf{u}=\mathrm{x} \mathbf{i}$, with $\mathbf{i}$ being the unit vectors in the global $\mathrm{x}$-coordinate directions, which yields

$$
\iiint_{D} \mathrm{dV}=\sum_{\mathrm{i}=1}^{\mathrm{N}} \iint_{S_{i}} \mathrm{xn} \mathrm{x} \mathrm{dA},
$$

where $\mathrm{n}_{\mathrm{x}}$ is the x-components of the unit normal vector of $S_{i} . \mathrm{V}$, the volume of $D$, is then obtained by 


$$
\mathrm{V}=\sum_{\mathrm{i}=1}^{\mathrm{N}} \iint_{S_{i}} \mathrm{xn} \mathrm{x} \mathrm{dA}
$$

If surface $S$ is discretized into 4-node quadrilateral patches, the integrands on the right side of Eq. (4) will be nonlinear functions, which in turn will require numerical integration. If triangular patches are used, the integrands will be linear. In DYNA3D, the surface is discretized into quadrilateral patches [1]. We then split a quadrilateral into two triangles to simplify the implementation and optimize the computation efficiency in the volume calculation. Due to the fact that $\mathrm{x}$ is a linear function and $\mathrm{n}_{\mathrm{x}}$ a constant over $S_{i}$, Eq. (4) can be rewritten as

$$
\mathrm{V}=\sum_{\mathrm{i}=1}^{\mathrm{N}} \overline{\mathrm{X}}_{\mathrm{i}} \mathrm{A}_{\mathrm{xi}}
$$

where $\overline{\mathrm{x}}_{\mathrm{i}}$ is the x coordinate of the centroid of triangular patch $S_{i}$, and

$$
\mathrm{A}_{\mathrm{xi}}=\mathrm{n}_{\mathrm{x}} \mathrm{A}_{\mathrm{i}}
$$

the projected area of triangular patch $S_{i}$ on the y-z plane.

$\overline{\mathrm{x}}_{\mathrm{i}} \mathrm{A}_{\mathrm{Xi}}$ in Eq. (5) is in fact the volume of the truncated triangular prism formed by $S_{i}$, its projection on the $\mathrm{y}-\mathrm{z}$ plane and the 3 line segments connecting the corresponding vertices. In essence, Eq. (5) states that the volume of $D$ is the summation of the volumes of all such prisms. For a triangular patch with an outward normal pointing away from y-z plane, its truncated prism has a positive volume, whereas the patch with normal pointing toward $y-z$ plane has a negative volume. When summed up, the negative prism volumes cancel the excess in the positive prism volumes to give the volume for $D$. Fig. 1 demonstrates the forming of the truncated prisms and the sign of their volumes.

In an explicit form suitable for implementation, Eq. (5) can be rewritten as

$$
\mathrm{V}=\sum_{\mathrm{i}=1}^{\mathrm{N}} \mathrm{V}_{\mathrm{i}},
$$

and

$$
\mathrm{V}_{\mathrm{i}}=\frac{1}{6}\left(\mathrm{x}_{1}+\mathrm{x}_{2}+\mathrm{x}_{3}\right)\left(\mathrm{y}_{1} \mathrm{z}_{2}+\mathrm{y}_{2} \mathrm{z}_{3}+\mathrm{y}_{3} \mathrm{z}_{1}-\mathrm{z}_{1} \mathrm{y}_{2}-\mathrm{z}_{2} \mathrm{y}_{3}-\mathrm{z}_{3} \mathrm{y}_{1}\right)
$$

$\mathrm{x}_{\mathrm{k}}, \mathrm{y}_{\mathrm{k}}$ and $\mathrm{z}_{\mathrm{k}}$, where $\mathrm{k}=1 \sim 3$, are the $\mathrm{x}-, \mathrm{y}$ - and $\mathrm{z}$-coordinate of node $\mathrm{k}$ of triangular patch $\mathrm{i}$ respectively. When choosing $\mathbf{u}=\mathrm{y} \mathbf{j}$ or $\mathbf{u}=\mathrm{zk}$, where $\mathbf{j}$ and $\mathbf{k}$ are the unit vector in $\mathrm{y}$ and $\mathrm{z}$ direction respectively, similar equations as Eq. (7b) with appropriate permutation of $\mathrm{x}, \mathrm{y}$ and $\mathrm{z}$ will be obtained. 


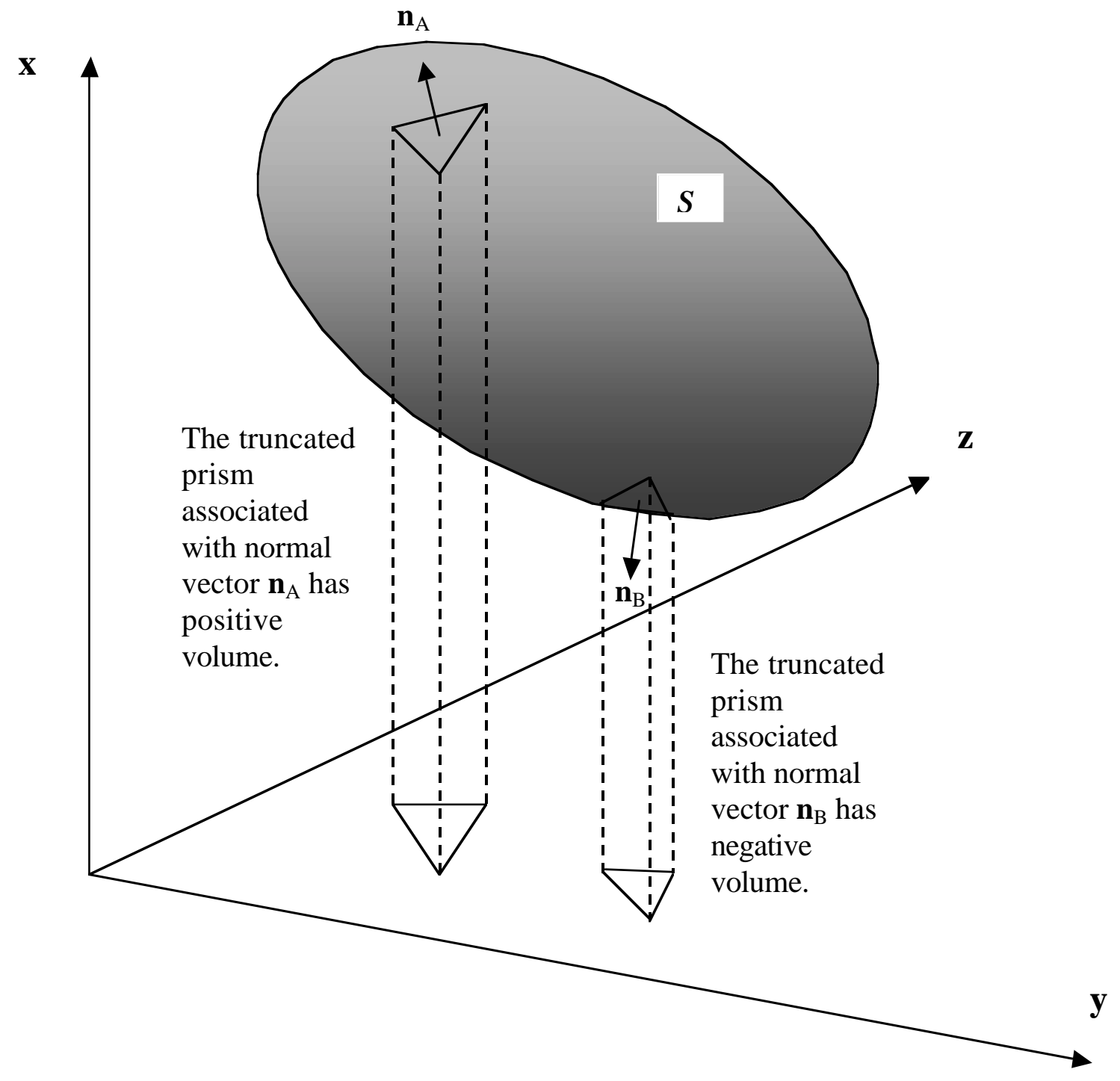

Figure 1. Truncated prisms formed by triangular patches on surface $S$ and their projections on the $\mathrm{y}-\mathrm{z}$ plane.

\section{Adaptive Patch Activation}

In some structure analyses, such as modeling a piston chamber or a gun barrel, the number of patches that enclose the chamber changes continuously. For example, when internal combustion causes a piston to move, fewer patches defining the chamber wall will be covered by the piston, which implies the volume increase. An adaptive technique is thus needed to determine the exposed patches automatically at any given time. 
For this purpose, we separate the surfaces enclosing a piston chamber into two categories: end surfaces and side surface. The end surfaces are the top of the piston and the end of the chamber opposite to the piston top, whereas the side surface represents the chamber sidewall. Different portion of the side surface would be covered by the piston at any given time, so the patches defining the surface are subject to be checked for exposure. Fig. 2 depicts the surfaces and demonstrates the check procedure.

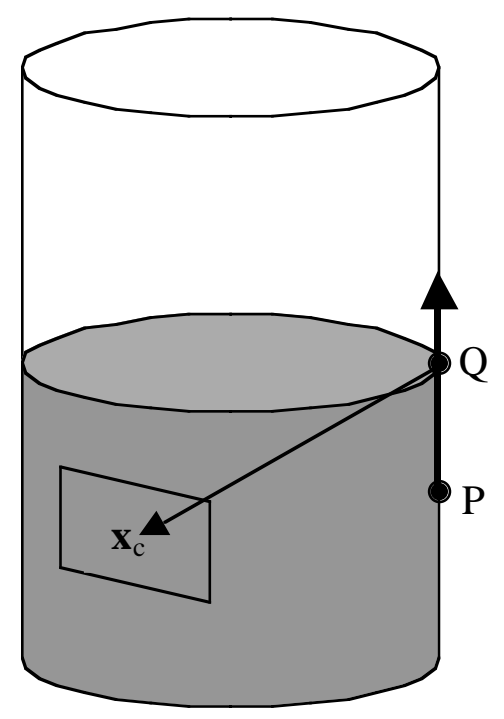

Figure 2. Procedure of defining an covered quadrilateral patch on the side wall of a piston chamber. The shaded cylinder represents the piston. The patch marked by the averaged location $\mathbf{x}_{\mathrm{c}}$ is covered by the piston because it is on the same side of the piston top surface with point $\mathrm{P}$.

Two nodes on the piston, $\mathrm{P}$ and $\mathrm{Q}$, are chosen to define the piston motion direction. For the exposure check, we look at a quadrilateral patch, instead of splitting it into two triangles, and compute the average location $\mathbf{x}_{\mathrm{c}}$ by

$$
\mathbf{x}_{\mathrm{c}}=\frac{1}{4} \sum_{\mathrm{k}=1}^{4} \mathbf{x}_{\mathrm{k}},
$$

where $\mathrm{k}=1,4$ represents the 4 nodes that constitute the patch. An imaginary plane perpendicular to $\overrightarrow{\mathrm{PQ}}$ is then drawn passing through point $\mathrm{Q}$. At a given time, if 


$$
\left(\mathbf{x}_{\mathrm{C}}-\mathbf{x}_{\mathrm{Q}}\right) \bullet \overrightarrow{\mathrm{PQ}} \leq 0
$$

which means the patch is located on the same side of the imaginary plane with point $\mathrm{P}$, this quadrilateral patch is deemed covered by the piston. Only patches not covered by the piston will be split into two triangles and take into account for the volume calculation. A quadrilateral patch is either fully counted or discounted in the volume calculation. No provision for partially exposed patch is available at this time.

\section{Projection Plane Selection}

Choosing the vector field $\mathbf{u}$ in Eq. (2) is equivalent to selecting a plane to project the enclosed chamber on. Any $\mathbf{u}$ with its divergence equal to a constant would serve the purpose. The distinct advantage of using $\mathbf{u}=\mathrm{xi}$, yj or zk is that the corresponding plane of projection is one of the coordinate planes. In Eq. (5), $\mathrm{A}_{\mathrm{xi}}$ is essentially the area of a triangle on the $\mathrm{y}-\mathrm{z}$ plane, involving only the $\mathrm{y}$ - and z-coordinates of its nodes. Had other linear $\mathbf{u}$ been chosen, the calculation of a triangular area in general 3-D space would have become necessary.

For a truly closed chamber, the selection of projection plane only affects the computation efficiency. However, if planes of symmetry are used in the model, the projection plane selection has its critical importance. When planes of symmetry cut through a closed chamber, the chamber is no longer closed and relies on the symmetry planes to enclose it. Because of the lack of discretized patches on the symmetry planes, the application of the Divergence Theorem would fail without some special care. In this case, the aim of the projection plane selection is such that the symmetry plane would have no contribution to the right hand side of Eq. (5). The obvious ways to achieve this are either take the plane of symmetry as the projection plane, which yields $\bar{x}_{i}=0$, or take a projection plane normal to the planes of symmetry, which makes, $\mathrm{A}_{\mathrm{xi}}=0$. In the event that two (2) planes of symmetry are used in the model, and they are not perpendicular to each other, a projection plane normal to both of the planes of symmetry must be chosen.

During our test of sample problems, the possible error accumulation due to an increasing $\bar{x}_{i}$ in Eq. (5) comes to light. The likely scenario for causing this is when the model going through large rigid body motion in an analysis. Numerical errors in evaluating equations such as Eq. (5) are inevitable and usually tolerable. However if the closed chamber is going through large displacement rigid body motion in the direction normal to the projection plane, the errors would be magnified, sometimes to an unmanageable level, because of the ever increasing $\bar{x}_{i}$ on the right hand side of Eq. (5). The end result is usually erroneous pressure changes.

To avoid this error magnification, a plane parallel to the selected coordinate plane and passing through a point on the enclosing surfaces is chosen as the plane of projection, instead of the coordinate plane. In essence, we let the plane of projection move along with the chamber in the pre-determined orientation. 


\section{Pressure Calculation and Application}

For every time integration step, all patches that constitute the closed chamber are first looped over for the summation of Eq. (5). Once the volume is obtained, a designated gas law can readily decide the pressure in the chamber. At this time, only a generalized ideal-gas power law

$$
\mathrm{p}=\mathrm{p}_{0}\left(\frac{\mathrm{V}_{0}}{\mathrm{~V}}\right)^{\mathrm{m}}
$$

is available. In Eq. (10), $\mathrm{P}_{0}$ and $\mathrm{V}_{0}$ are the initial pressure and volume respectively. $\mathrm{m}$ is the gas law constant. User must provide $\mathrm{P}_{0}$ and $\mathrm{m}$, but $\mathrm{V}_{0}$ is calculated by the code in the beginning of the analysis. Other gas laws can be integrated into the code easily when they are needed.

Pressure $\mathrm{P}$ will then be applied to each patch that forms part of the closed chamber in the direction normal and pointing to the patch. In the case of a piston chamber, pressure will only be applied fully to patches that participated in the chamber volume calculation. The pressure is then multiplied by the a patch's area and equally distributed to its four (4) nodes.

\section{DYNA3D User Input Formats}

Users must use the DYNA3D Free Format Keyword Option [1] to activate these features. They are grouped into two categories: closed_volume and piston_chamber. In general, these two features must be associated with DYNA3D Load Curve, which in turn binds a list of 4-noded quadrilateral patches together to define the enclosing surface. closed_volume takes a single Load Curve to define the enclosing surface: whereas piston_chamber takes two, since its sidewall and end surface are labeled distinctively. For the closed_volume, the input sequence is

closed_volume gas_law cutoff_opt lcv $\mathrm{v}_{0} \mathrm{~m}$ plane,

where

gas_law : designates the gas law for pressure calculation.

=ideal_gas is the only available choice at this time.

Cutoff_opt : designates the gas pressure cutoff option.

= cutoff means only positive pressure will be applied and negative pressure is cutoff. Positive pressure is the pressure pointing toward the patch in its normal direction.

=no_cutoff means no negative pressure cutoff.

Icv : 4-noded quadrilateral Pressure Load patches associated with Load Curve number 1 cv form this closed volume. 


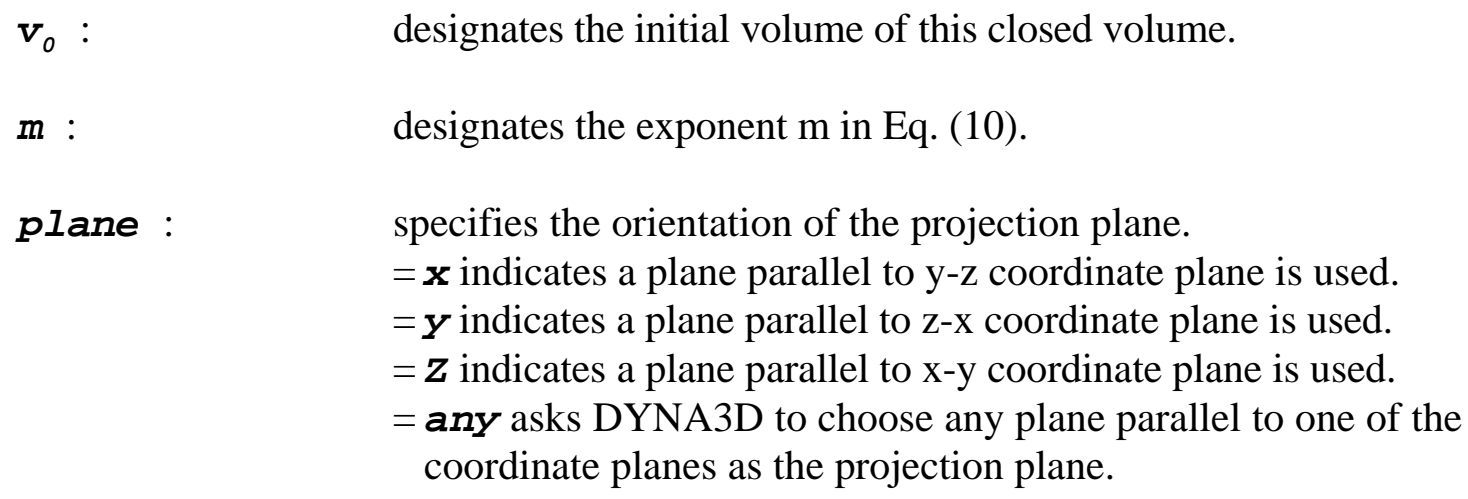

For piston_chamber, the input sequence is

piston_chamber gas_law cutoff_opt lcv1 lcv2 $v_{0} m$ plane $P Q$,

where

gas_law : designates the gas law for pressure calculation.

=ideal_gas is the only available choice at this time.

Cutoff_opt : designates the gas pressure cutoff option.

= cutoff means only positive pressure will be applied and negative pressure is cutoff. Positive pressure is the pressure pointing toward the patch in its normal direction.

= no_cutoff means no negative pressure cutoff.

1cv1 : $\quad$ 4-noded quadrilateral Pressure Load patches associated with Load Curve number $1 \mathbf{c v} 1$ form the end surfaces for this piston chamber.

1cv2 : $\quad$ 4-noded quadrilateral Pressure Load patches associated with Load Curve number $1 \mathbf{c v} 2$ form the sidewall for this piston chamber.

$\boldsymbol{v}_{0}: \quad$ designated the initial volume of this closed volume.

$\boldsymbol{m}: \quad \quad$ designated the exponent $\mathrm{m}$ in Eq. (10).

plane: $\quad$ specifies the orientation of the projection plane.

$=\mathbf{x}$ indicates a plane parallel to $\mathrm{y}-\mathrm{z}$ coordinate plane is used.

$=\boldsymbol{y}$ indicates a plane parallel to $\mathrm{Z}$ - $\mathrm{x}$ coordinate plane is used.

$=\boldsymbol{Z}$ indicates a plane parallel to $\mathrm{x}-\mathrm{y}$ coordinate plane is used.

= inclined indicates the use of a plane parallel to a coordinate plane whose normal vector aligns closest to the vector $\overrightarrow{\mathrm{PQ}}$. 
$P$ :

$Q$ : specifies the node number for the node serves the purpose of point $\mathrm{P}$ in Fig. 2.

specifies the node number for the node serves the purpose of point $\mathrm{Q}$ in Fig. 2.

\section{Examples}

A sample problem with a plane of symmetry as shown in Fig. (3) is used to validate the algorithm and its implementation into DYNA3D. The darker cylinder surface is the piston sidewall that is subject to the exposure check. Two surfaces, marked by the same Load Curve number in DYNA3D, are fitted in to the cylinder. The Load Curve number assigned to these two surfaces must not be the same as the Load Curve number given to the sidewall so that the exposure check can be limited to the sidewall patches. The lower surface moves closer to the upper surface at a constant net velocity of $0.14 \mathrm{in} / \mathrm{sec}$. The diameter of the cylinder is 7 inches, and the positive $\mathrm{z}$ direction points upward. Only half of the model is presented because of symmetry.

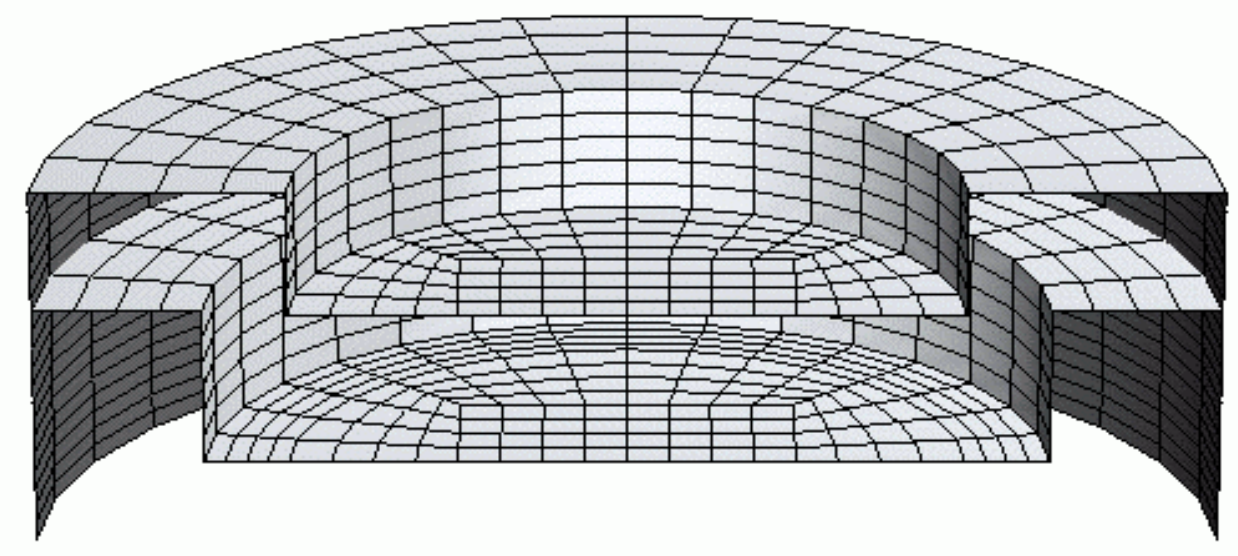

Figure 3. A sample problem for piston chamber pressure calculation. 
The pressure-volume relation follows Eq. (10) with $\mathrm{m}=1$. The initial pressure is set as $\mathrm{p}_{0}=1.0$ $\mathrm{psi}$, and the initial chamber volume is $20.06 \mathrm{in}^{3}$. The analytic solution for the pressure is

$$
\mathrm{p}=\frac{\mathrm{p}_{0} \mathrm{~V}_{0}}{\mathrm{~V}_{0}-\frac{1}{2} \pi \mathrm{r}^{2} \mathrm{vt}}
$$

where $\mathrm{v}$ is the constant closing velocity between the two end surfaces, $\mathrm{r}$ is the cylinder diameter and $t$ the time.

A plane parallel to the $\mathrm{x}-\mathrm{y}$ coordinate plane was chosen as the projection plane in DYNA3D analysis. As can be seen in Fig. 4, a close agreement between the numerical solution by DYNA3D and the analytic solution is evident. Other structures with gas-filled chambers are tested as well. Because of the sensitive nature of the problems, the results are not presented here. They would have to be presented in a more restrictive forum.

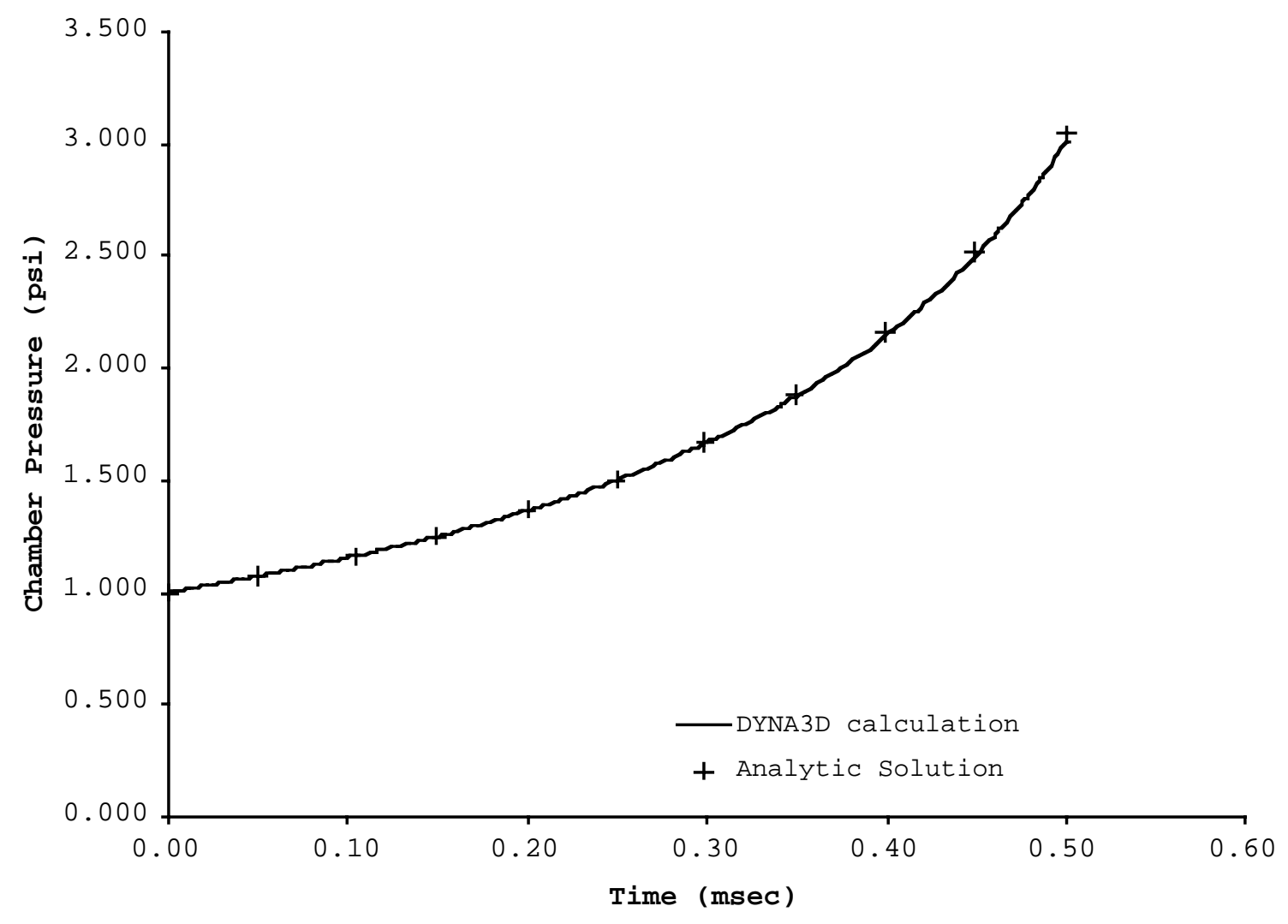

Figure 4. The pressure time history for the sample piston chamber. 


\section{Conclusions and Future Work}

The proposed algorithm for closed-volume pressure calculation has been successfully applied to the Weapons Program. The algorithm has provided sufficiently accurate pressure prediction at a reasonable computational cost in several analyses. Applications in other areas, such as engine design and airbag analysis, are definitely possible.

Two critically important principles in using this feature are: (a) the chamber must be entirely enclosed by either discretized patches or boundary planes; and (b) the proper selection of projection plane so that the boundary planes have no contribution in volume calculation.

In anticipation of broadening application of this feature to problems of larger size, we need to complete the parallel implementation for ParaDyn in the near future. Additional pressurevolume equations will also be added as needed. 


\section{References}

[1] Current Developer: Lin, J.I., "DYNA3D: A Nonlinear, Explicit, Three-Dimensional Finite Element Code for Solid and Structural Mechanics - User Manual", University of California, Lawrence Livermore National Laboratory (1993), UCRL-MA-107254.

[2] Kreyszig, E., “Advanced Engineering Mathematics: Third Edition” ( John Wiley \& Sons), p. 354. 


\section{Acknowledgement}

The authors wish to thank Dr. Gregory Kay and Dr. Edward Zywicz for their helpful discussion and input on this subject. Dr. Kay also provided some of the sample problems. 